International Journal of Distributed and Parallel Systems (IJDPS) Vol.3, No.1, January 2012

\title{
ENERGY EFFICIENT AND QOS AWARE ANT COLONY OPTIMIZATION (EQ-ACO) Routing PRotocol For WIRELESS SENSOR NETWORKS \\ Devee Prasan. $U^{1}$, Murugappan. $S^{2}$
}

\author{
${ }^{1}$ Dept of CSE, Aditya Institute of Technology and Management, Tekkali, Srikakulam, \\ Andhra Pradesh \\ ${ }^{2}$ Department of Computer science \& Engineering, Annamalai University \\ udeveeprasan@gmail.com, drmryes@gmail.com
}

\begin{abstract}
Wireless sensor network (WSN) is a collection of autonomous, tiny, large number of densely deployed sensor nodes; these sensor nodes are smart, effective which offers very powerful and versatile networking where traditional wired and wireless networking is unable to deploy.
\end{abstract}

\section{KEYWORDS}

Wireless Sensor Network, Routing, Energy Efficient, Qos Aware, Ant Colony Optimization.

\section{INTRODUCTION}

WSN is applied widely in many engineering applications like monitoring insecure and remote borders, movements of terrorist in the high forest area, monitoring LPG pipes laid in deep underwater like sea. There are many research issues are identified and solutions are proposed in the past few years which includes optimum routing protocol, medium access control, transport control protocol, scalability, congestion and packet loss recovery.

For the improvement of modern and advanced technological world, the computers are contributed more optimality and ease of implementation. Especially the computer networks provide more dominant and powerful way for resource sharing and data transmission. The computer network starts with wired technologies and spreads its wings using wireless components and now it advanced using sensor elements. The collection of sensor nodes by enabling cooperation, coordination and collaboration among sensor nodes is formed Wireless Sensor Network (WSN); the WSN consists of multiple autonomous nodes with a base station. The base station is used for collecting information from the sensor nodes which may be mobile or fixed. The sensor node identifies or monitoring the activity or events in the environment or region of the network, and transmits it to the base station.

Sensor nodes are fitted with an on-board processor, which has following characteristics:

- Low processor and bus clock speeds,

- Low sizes of RAM and Flash memory, and

- Lesser wireless bandwidths. 
International Journal of Distributed and Parallel Systems (IJDPS) Vol.3, No.1, January 2012

\subsection{Hardware and Software Components of WSN}

The recent research in the WSN has led to significant improvements in the characteristics of the wireless sensors. Crossbow Technology which is developed in collaboration with the contribution of the researchers in University of California Berkeley is a wide spread open source development, and the Crossbow Technology is also distributed commercially with advanced wireless sensors. The first commercial generation wireless sensor developed by them was named as Rene Mote; which designed with the next generation wireless sensors called MICA sensors. The basic MICA hardware available commercially is a square inch in size and consumes a fraction of a watt of power.

The hardware design of a MICA sensor mote consists of a small, low-power radio and processor board known as a Mote Processor/ Radio (MPR board) and one or more sensor boards known as a Mote Transmission Sensor (MTS, board). The combination of these two types of boards forms a networkable wireless sensor. For example, the present sensor of Rene Mote has ATMEL ATMEGA processor family, with speeds of up to $8 \mathrm{MHz}, 128 \mathrm{~KB}$ of flash memory and $4 \mathrm{~KB}$ of SRAM. The MPR consists of a radio as its major component, which is used to communicate with other sensors as well as the base station. The radio for MICA consists of a $916 \mathrm{MHz}$ ISM band transceiver, antenna, and collection of discrete components to configure the physical layer characteristics, such as signal strength and sensitivity. The radio operates at a data speed of $50 \mathrm{Kbps}$ and operates in an ON/OFF key mode.

The radio can operate in three modes such as transmit, receive, or power-off mode. Wireless sensors thus make frequent transitions from active to sleep state in order to conserve energy. In active state, the sensor nodes perform the task of receiving messages from neighbouring nodes as well as observing an event. The sensor nodes move to the sleep state when there is no work to be performed in order to conserve energy. The sensors in sleep state move into active state upon occurrence of an event. In the current sensors the sleep mode also increases more than one state, for example, the MICA sensor has three sleep modes such as idle mode, power down mode and power save mode. The idle mode just shuts off the processor; the power down mode shuts down all resources except event handler, the power save mode is similar to power down except that it doesn't stop the asynchronous timer.

The software for the motes is developed on the Tiny OS platform. Tiny OS is an efficient and modular embedded software platform for the Motes. It is a component-based runtime environment designed to provide support for deeply embedded systems that require intensive concurrent operations while being constrained by minimal hardware resources. Tiny OS has been developed on an open source software platform by UC Berkeley with active support from a large community of users. Tiny DB is a query-processing system for extracting information from a network of Tiny OS sensors.

\subsection{Design of WSN}

The sensors are deployed in an environment as large in numbers and the density of the nodes are defined in the equation 1 ,

$$
\mu(R)=\left(N \bullet \pi \cdot R^{2}\right) / A--(1)
$$

where, $N$ is the number of scattered sensor nodes in region $\mathrm{A} ; \mathrm{R}$, the radio transmission range; $\mu(R)$ gives the average number of nodes within the transmission radius of each node in $\operatorname{region} A$.

The entire networks of sensor nodes are divided into many sub-network or clusters based on their geo based physical location. The groups which may be sub-network or clusters that consists of nodes close to each other having lower communication costs and lesser energy for transmission and when two nodes are far away from each other, then there is no any common information and transmission. The clusters or sub-network are grouped using various location discovery methods. The sensors nodes will operate in either a synchronous or an asynchronous 
manner. The sending and receiving processes synchronize every message in a synchronous system and the sending process works independently of the receiving process in an asynchronous system. The asynchronous system has lower communication costs and operates in a non-blocking manner; therefore, most of the WSN applications like to operate in an asynchronous system. There are many research issues in the WSN, this paper further concentrates in the routing issues.

The most important criteria for the wireless sensor network are the longevity of operation that the wireless sensors should function correctly for a long period of time after their initial deployment. The wireless sensors will derive energy from their in-built batteries, and in general all batteries usually have a fixed lifetime. Therefore it is desirable that the power consumption of the nodes should be minimized. Now a day, rechargeable batteries can also be employed for wireless sensors, they are attached to a solar or photovoltaic cell and this has limited application. The photovoltaic cells, however, produce a limited amount of power and therefore the energy must still be conserved. Communication costs account for the major amount of power consumption, the ratio of power consumption for communication to computation is often estimated as 1000:1. Decreasing the amount of communication between nodes can reduce the energy consumption and thus increase the life of the sensor network. A lot of research has therefore concentrated on minimizing the amount of communication taking place in the wireless sensor network.

\subsection{Underwater WSN}

The WSN have been used in many applications such as monitoring radiation and nuclearthreat detection systems, weapon sensors for ships, biomedical applications, habitat sensing, seismic monitoring, military sensing, physical security, air traffic control, traffic surveillance, video surveillance, industrial and manufacturing automation, process control, inventory management, distributed robotics, weather sensing, environment monitoring, national border monitoring, and building and structures monitoring. The detailed application of WSN includes Forest fire detection, Flood and Tsunami detection and assessment, Tracking and monitoring doctors and patients inside a hospital, Vehicle tracking and detection. Most of the research in the WSN is focused for military applications, In Military applications the WSN are deployed for Monitoring terrorists, Monitoring their own military forces and equipment, battlefield surveillance, targeting the attack area, for the assessment of battle damage. Now a day, the WSN is deployed for monitoring HVAC, cooking devices and more commercial in home applications.

This paper concentrates the underwater WSN; the underwater wireless Sensor networks (UWSN) are constituted by sensors and autonomous underwater vehicles (AUV). The typical underwater architecture is shown in the figure 1, the photocopy of real AUV is shown in figure 2 and the implementation of AUV in underwater is shown in figure 3 (MIT-Cambridge; CSIRO ICT-Australia). AUV is a device used to coordination, information sharing and interact to perform underwater monitoring. Most of the architectural design, functionality, computation and other resource constraints like limited energy of underwater implementation are similar to ground based WSN. The characteristics of UWSN are

- The quality of radio waves propagated in the UWSN is less due to the high energy absorption of water. Therefore, underwater communications are based on acoustic links characterized by large propagation delays. The propagation speed of acoustic signals in water (typically $1500 \mathrm{~m} / \mathrm{s}$ ) is five orders of magnitude lower than the radio wave propagation speed in free space.

- Acoustic channels have low bandwidth. 
International Journal of Distributed and Parallel Systems (IJDPS) Vol.3, No.1, January 2012

- The link quality in underwater communication is severely affected by multipath, fading, and the refractive properties of the sound channel.

- The bit error rates of acoustic links are often high, and losses of connectivity arise.

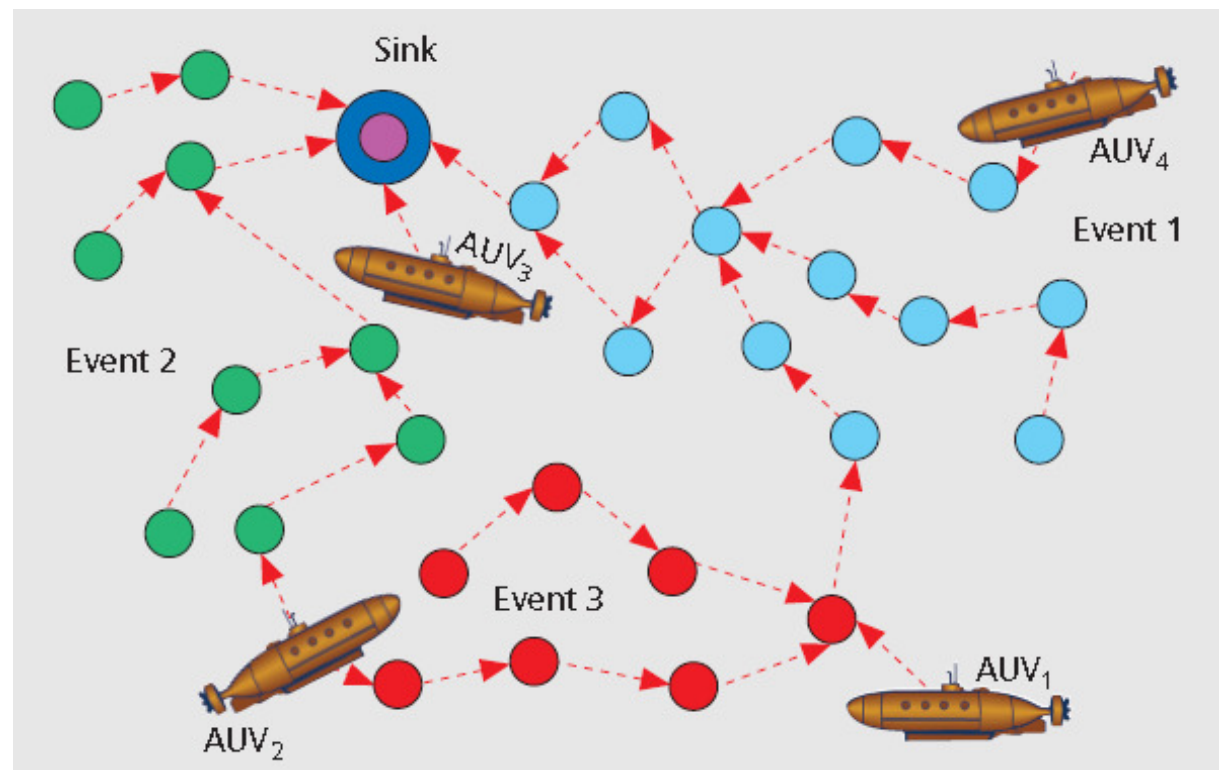

Figure 1. Typical underwater WSN and multipath communication

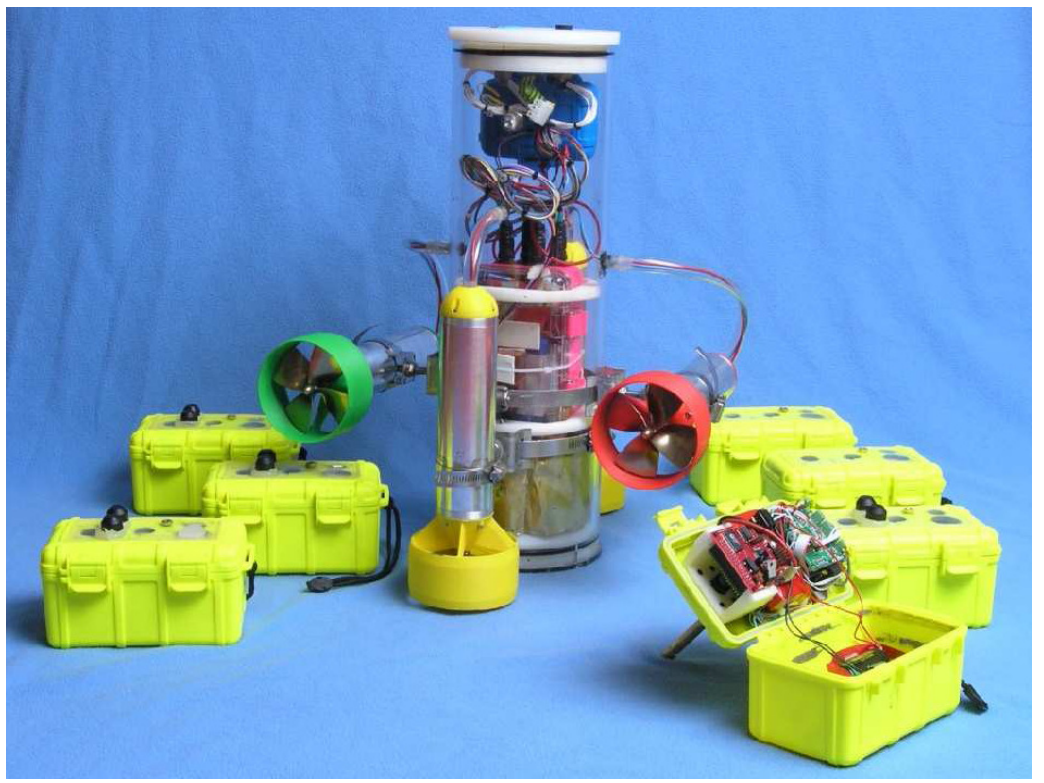

Figure 2. Sample AUV used in underwater WSN 
International Journal of Distributed and Parallel Systems (IJDPS) Vol.3, No.1, January 2012

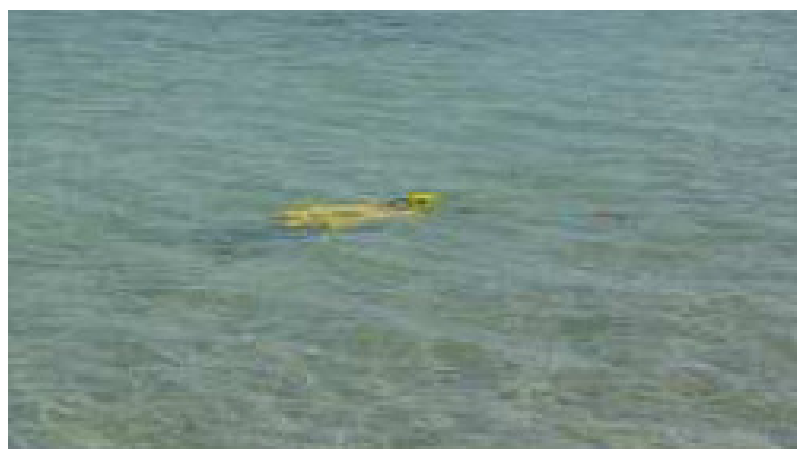

Figure 3. AUV in underwater WSN

\subsection{Overview of Routing protocol in WSN}

The energy requirements and limited resources like lesser memory of sensor nodes are the major important deciding factor for designing routing protocol for WSN. Let, there are $p$ intermediate nodes from the region to the base station, $m$ nodes in the group, and each message communication requires $q$ micro-joules of energy, then the multi-hop method entails transmission of information from each node to the base station requires $p^{*} m^{*} q$ micro-joules of energy. Reducing this energy requirement for each communication is the major task in WSN and it can be reduced by increasing number of node deployed in the groups. But the numbers of deployed nodes are increased then the initial cost of the system will go high. Therefore, a tradeoff is required to balance the initial cost as well as lesser transmission energy. This trade-off is possible by an optimal routing algorithm. The routing protocols in WSN can be classified as four major types, such as Data centric routing, Location based Routing, Hierarchical routing and QoS oriented routing.

In the Data centric routing protocols such as Constrained Anisotropic Diffusion Routing (CADR), COUGAR, ACQUIRE, Gradient Based Routing (GBR), Directed Diffusion Rumor Routing, Sensor Protocols for Information via Negotiation (SPIN), the sink sends queries to certain WSN regions and waits for data from the regions selected. Because data are being requested through queries, attribute-based naming is necessary to specify the properties of data. Due to the large number of nodes deployed, in many WSNs it is not practical to assign global identifiers to each node. This, along with potential random deployment of Wireless Nodes, makes it challenging to select a specific (or a specific set of) nodes to be queried. Hence, data are typically transmitted from every nodes with in the deployment region; this gives rise, however, to significant redundancy along with inefficiencies in terms of energy consumption. Therefore, it is desirable to have routing protocols that will be able to select a set of sensor nodes and utilize data aggregation during the relaying of data. This has led to the development of datacentric routing.

Energy adaptive clustering hierarchy (LEECH), Threshold sensitive energy efficient sensor network protocol (TEEN), Adaptive TEEN (APTEEN), Power Efficient Gathering in Sensor Information System (PEGASIS) are few Hierarchical routing protocols. In which, a single-tier network, may be the gateway or the cluster-head, can cause the gateway node to become overloaded, particularly as the density of sensors increases. This, in turn, can cause latency in event status delivery. To permit WSNs to deal with a large population of nodes and to cover a large area of interest, multipoint clustering has been proposed. The goal of hierarchical routing is to manage the energy consumption of nodes efficiently by establishing multi-hop communication within a particular cluster, and by performing data aggregation and fusion to 
decrease the number of transmitted packets to the sink. The multi path routing of WSN is shown in figure 4.

Location information about the nodes can be utilized in the location based routing in which the data is transmitted in an energy- efficient manner. The location information is used to calculate the distance between and small two given nodes so that energy consumption can be determined (or at least, estimated). Minimum Energy Communication Network (MECN), Small MECN (SMECN), Geographic Adaptive Fidelity (GAF) are few location based routing protocols.

The QoS-oriented routing protocos offers Quality of service (QoS) aware protocols which consider end-to-end delay requirements in setting up the paths in the sensor network, Sequential Assignment Routing (SAR), Stateless protocol for end-to-end delay (SPEED) are few examples for this routing protocol. Chandramathi et al (2007) presents energy aware routing for WSN using traditional models. Anitha et al (2011) presents the performance evaluation of adhoc sensor nodes.

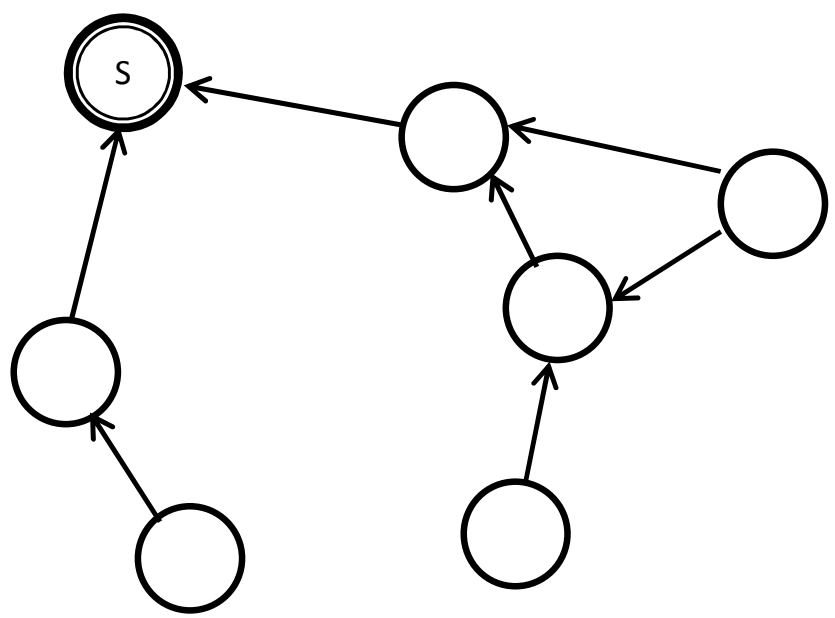

Figure 4. Multi path communication in WSN, in which the S represents Sink

The Routing in the UWSN is critical due to the large propagation delays, the low bandwidth, the difficulty of battery refills of underwater sensors, and the dynamic topologies. Therefore, routing protocols should be designed to be energy-aware, robust, scalable and adaptive.

\section{Proposed EQ-Aco}

The Ant Colony Optimization (ACO) is a swarm intelligence technique which is applied many engineering optimization problems. Swarm intelligence (SI) is a new discipline of study that contains a relatively optimal approach for problem solving which is the imitation inspired from the social behaviours of insects and of other animals, for ex: ACO, artificial bee colony algorithms and fire fly algorithm. The ACO is an optimization technique which is widely applied for a variety of optimization problems and in almost all engineering field of studies. Chandramohan et al $(2008,2010,2011)$ reviewed ACO and its applications in the various engineering field of study and proposes ACO for wired and wireless routing. In this paper, the ACO is proposed for QoS aware routing protocol. 
International Journal of Distributed and Parallel Systems (IJDPS) Vol.3, No.1, January 2012

\subsection{Overview of existing ACO}

The field of "Ant Algorithm" studies models derived from the observation of real ants' behaviour, and uses these models as a source of inspiration for the design of novel algorithms for the solution of optimization and distributed control problems. The main idea is that the selforganizing principles which allow the highly co-ordinated behaviour of real ants can be exploited to co-ordinated populations of artificial agents that collaborate to solve computational problems. Several different aspects of the behaviour of ant colonies have inspired different kinds of ant algorithms, for ex: foraging, division of labour, brood sorting, and co-operative transport. One of the most successful examples of ant algorithms is known as "Ant Colony Optimization (ACO)".

Suppose that there are four ants $\left(A_{1}, A_{2}, A_{3}\right.$, and $\left.A_{4}\right)$ and two routes (R1 and R2) leading to a food source $\left(F_{0}\right)$, where $R_{1}$ and $R_{2}$ such that $R_{1}>R_{2}$ and $R_{1}=2 * R_{2}$. Initially, all ants are at the decision point $N_{e}$ and they have to select between $R_{1}$ and $R_{2}$ to reach $F_{0}$.

1) At $\mathrm{N}_{\mathrm{e}}$, all ants have no knowledge about the location of food $\left(\mathrm{F}_{0}\right)$. Hence, they randomly select from $\left\{R 1, R_{2}\right\}$. Suppose that $A_{1}$ and $A_{2}$ choose $R_{1}$, and $A_{3}$ and $A_{4}$ choose $R_{2}$.

2) $\quad A_{s} A_{1}$ and $A_{2}$ move along $R_{1}$, and $A_{3}$ and $A_{4}$ move along $R_{2}$, they leave a certain amount of pheromone along their paths $\tau_{\mathrm{R} 1}$ and $\tau_{\mathrm{R} 2}$, respectively.

3) Since $R_{1}>R_{2}, A_{3}$ and $A_{4}$ reach $F_{0}$ before $A_{1}$ and $A_{2}$. When $A_{3}$ and $A_{4}$ pass $R_{2}$ to reach $F_{0}$, $\tau_{R 2}=2$, but $A_{1}$ and $A_{2}$ have yet to reach $F_{0}$ and $\tau_{R 1}=0$. To return to $N_{e}$ from $F_{0}, A_{3}$ and $A_{4}$ have to choose between $R_{1}$ and $R_{2}$. At $F_{0}, A_{3}$ and $A_{4}$ detect that $\tau_{R 2}>\tau_{R 1}$, hence they are more likely to select $\mathrm{R}_{2}$.

4) $\quad A_{s} A_{3}$ and $A_{4}$ pass $R_{2}$ for the second time to reach $N_{e}, \tau_{R 2}$ is incremented to 4 . The increase in $\tau_{R 2}$ further consolidates $R_{2}$ as the shorter path. When $A_{1}$ and $A_{2}$ reach $F_{0}, \tau_{R 2}=4$ and $\tau_{R 1}$ $=2$. Hence, $A_{1}$ and $A_{2}$ are more likely to select $R_{2}$ to return to $N_{e}$.

In this example, any ant at $\mathrm{F}_{0}$ (respectively, $\mathrm{N}_{\mathrm{e}}$ ) will be able to determine the optimal path once $\mathrm{A}_{3}$ and $\mathrm{A}_{4}$ reach $\mathrm{F}_{0}$. If an ant is at a choice point when there is no pheromone (e.g., initially at $\mathrm{N}_{\mathrm{e}}$ ), it makes a random decision with a probability of 0.5 of choosing $\mathrm{R}_{1}$ or $\mathrm{R}_{2}$. However, when pheromone is present (e.g., when the ant is at $\mathrm{F}_{0}$ ), there is a higher probability that it will choose the path with the higher concentration of pheromone.

\subsection{Detailed Description of EQ-ACO:}

When sensor nodes are deployed in underwater sensing such as monitoring LPG pipelines, a QoS aware routing is offers optimality. Therefore this paper concentrates on QoS aware routing. In the QoS aware routing the trade-off between guarantee of QoS of the network and the lifetime of the network is a critical issue. i.e., the performance of QoS aware routing and the performance of Energy efficient routing are inversely proportional to each other. For example, the multi path routing is provides higher QoS but it leads to more energy loss. Even though the energy is lost more, the QoS aware routing is an optimal choice for monitoring LPG like pipelines laid in the underwater. Because the small lose in the data may leads to heavy loss for the system, therefore the underwater WSN may be termed as hard real time WSN system.

Most of the existing routing proposed in WSN is based on a flat and homogenous architecture, in which every sensor nodes has an identical physical capabilities and a sensor node can interact only with its neighbouring sensors. However, flat topologies are not always best suited to handle the amount of traffic generated by multimedia applications, including audio and video. And in the underwater WSN, the location based routing also not suitable. 
The single path routing algorithms are simpler and they consume less energy whereas the multipath routing is more reliable than single path. The reliability can be characterized by way of a packet delivery ratio, which is defined as the ratio between the numbers of unique packets successfully received by the sink over the number of packets generated by source nodes. In general, the multipath routing offers highly reliable data delivery in wired, wireless and WSN. This is due to instead of single copies of information, multiple copies of data along different paths are sent to reduce data delivery delays by sharing transmission delays among the different paths available from the source to the destination as well as avoiding data loss in the network.

In heterogeneous WSNs, a wireless node may also contain different sensors such as audio, video and scalar sensors. As the priority of such heterogeneous traffic differs, considering service differentiation appears to be an additional issue. There are two types of traffic: real-time traffic has hard time constraints, such as delays, although it is more tolerant of packet losses; non-realtime traffic usually exists in networks and produces thousands of packets which are generated synchronously or asynchronously. In the proposed work, to achieve QoS as well as energy efficiency, an additional parameter called Node-to-Sink Energy (NSE) is introduced.

The problem of determining a QoS route that satisfies multiple constraints is known to be NP-hard. The problem definition requires exact information regarding nodes on their paths to the destination, which is almost impossible to obtain in WSNs. Hence, to solve this challenge, each source node periodically collects information from its one-hop neighborhood. One-hop link metrics are much easier to acquire.

Given the tuple $\left(D_{\text {req }}, R_{\text {req }}\right)$ of QoS requirements specified by the source node, that represents the Node-to-Sink delays $\left(\mathrm{D}_{\text {req }}\right)$ and the reliability level $\left(\mathrm{R}_{\text {req }}\right)$. To find the set of paths $\xi\left(S_{0}, S_{d}\right)$ from the source node ' $S_{o}$ ' to the destination node ' $S_{d}$ ' that maximizes the NSE, subject to deadline and reliability constraints which represents in the equation 2.

$$
\max \mathrm{B}_{\mathrm{So}, \mathrm{Sd}}(\xi)-(2)
$$

Subject to:

$$
\begin{aligned}
& \mathrm{D}_{\text {So, Sd }}(\xi) \leq \mathrm{D}_{\text {req }}-(3) \\
& \mathrm{R}_{\text {So, Sd }}(\xi) \leq \mathrm{R}_{\text {req }}-(4)
\end{aligned}
$$

The equation 2, maximizes the residual energy of the selected nodes, while constraints expressed in the equation 3 and 4 shows the delay and reliability requirements of the data source node, respectively.

In every time interval T(i), the forward ants are generated and flooded in the WSN from the each sensor node to sink with the forward set is assumed as null. The forward ant is a tiny control packet like hello protocol in the traditional network.

The forward ant which is forwarded towards clusters of sensor nodes and collects information on each nodes. The candidate set is updated based on the information on each node it visits,

$$
C(i)=\left\{\begin{array}{l}
L_{i j} / H_{j} \\
D_{i}+D_{i j}
\end{array}-(5)\right.
$$


Where $\mathrm{C}(\mathrm{i})$ is a candidate set of node $\mathrm{i}, L_{i j}$ is the cumulative link delay from node to sink in ms, $\mathrm{H}$ is the hop count in numbers, $\mathrm{D}$ is the delay due to traffic in the node ' $\mathrm{i}$ ' or cumulative delay between the nodes 'ij'.

The forward ant reaches sink with Candidate set information and the sink receives multiple set of such candidate key. From the candidate key, NSE is computed using equation 6.

$$
\mathrm{NSE}(\mathrm{p})=\mathrm{C}(\mathrm{i}, 1) * \mathrm{~N}(\mathrm{p})+\mathrm{C}(\mathrm{i}, 2) / \mathrm{N}(\mathrm{p})-(6)
$$

Where $\mathrm{N}(\mathrm{p})$ is the number of nodes in the concern path ' $\mathrm{p}$ '.

Suppose the sink receives many candidate key from a node 'i', then the NSE of each path is calculated. The lowest NSE is approved as main path and the next lowest NSE is approved as auxiliary path 1 . Similarly the auxiliary path 2 to $n-1$ is also ranked in the sink.

The backward ant is generated when each forward ant is received in the sink, and the backward ant is travelled towards concern node in the same path in which the forward ant is travelled. This route information is stored in the sink, as the sensor node contains limited memory, the route information is not possible to store in each sensor node.

\section{Results And Performance Analysis}

The Simulation environment is described here:

a) Best Case:

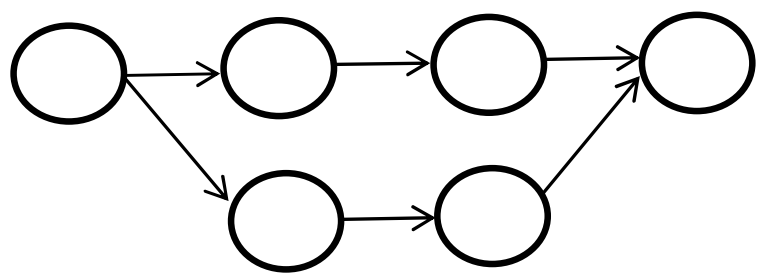

In this case, there are only two paths are available from sensor node ' $\mathrm{i}$ ' to sink, which is shown in the figure 5 .

Figure 5. Architecture of Best case

b) Worst Case:

In this case, there are only one path is available from sensor node ' $\mathrm{i}$ ' to sink, which is shown in the figure 6. 
International Journal of Distributed and Parallel Systems (IJDPS) Vol.3, No.1, January 2012

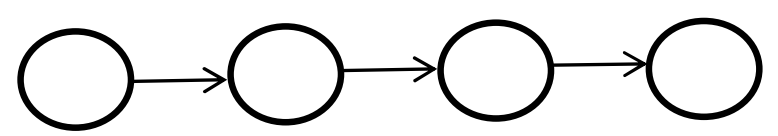

Figure 6. Architecture of Worst case

c) Average Case:

In this case, there are many paths are available from sensor node 'i' to sink.

The QoS is calculated in the three cases described in the above, in terms of reliability and link delay.

The proposed work is tested for on time packet delivery ratio, total number of packet delivery, and average packet delay which is shown in the figure 7,8 and 9.

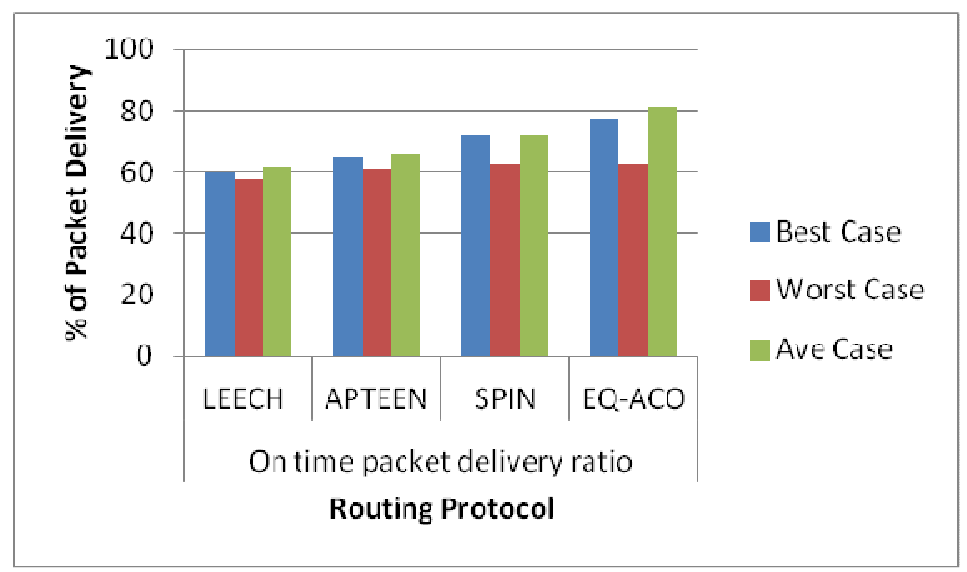

Figure 7. On time packet delivery ratio

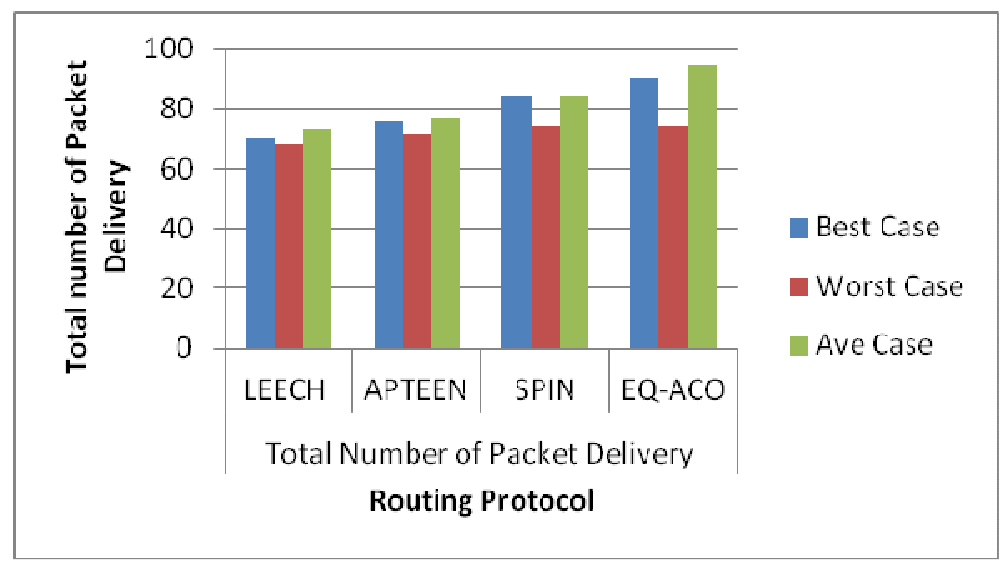

Figure 8. Total number of packet delivery 
International Journal of Distributed and Parallel Systems (IJDPS) Vol.3, No.1, January 2012

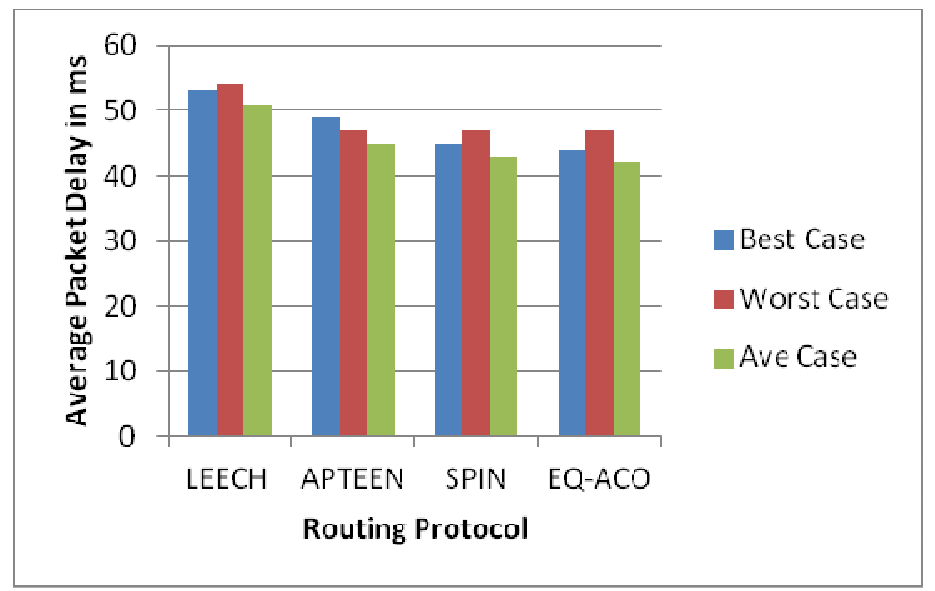

Figure 9. Average Packet Delay

\section{CONCLUSION}

The performance of ACO is optimal in many enginering applications, it is proved once again in the routing issues of WSN. The results of the poposed work shows optimality in all aspects, and offers energy efficient routing as well as QoS aware routing.

\section{REFERENCES}

[1] Anitha and Chandrasekar, 2011. Comparative Performance Evaluation of Routing Algorithms in IEEE 802.15.4 and IEEE 802.11 with Different Ad Hoc Routing Protocol. Journal of Computer Science, 7: 731-735. DOI: 10.3844/jcssp.2011.731.735.

[2] Chandra Mohan, B. and Baskaran, R. 2010, Improving network performance by optimal load balancing using ACO based Redundant Link Avoidance algorithm, International Journal of Computer Science Issues, 7, 3, : 27-35

[3] Chandra Mohan, B. and Baskaran, R. 2011, Reliable Transmission for Network Centric Military Networks, European Journal of Scientific Research, 50, 4: 564-574

[4] Chandra Mohan, B. and Baskaran, R. 2011, Reliable Barrier-free Services in Next Generation Networks, Lecture Notes in Computer Science, Second International Conference on Advances in Power Electronics and Instrumentation Engineering, PEIE 2011, Springer-Verlag Berlin Heidelberg, CCIS $148: 79-82$

[5] Chandra Mohan, B. and Baskaran, R. 2011, Survey on Recent Research and Implementation of Ant Colony Optimization in Various Engineering Applications, International Journal in Computational Intelligent Systems, 4, 4:566-582

[6] Chandra Mohan, B. and Baskaran, R. 2011, Energy Aware and Energy Efficient Routing Protocol for Adhoc Network using Restructured Artificial Bee Colony System, HPAGC 2011, Springer-Verlag Berlin Heidelberg, CCIS 169 : 480-491

[7] Chandra Mohan, B., Sandeep, R. and Sridharan, D. 2008, A Data Mining approach for Predicting Reliable Path for Congestion Free Routing using Self-Motivated Neural Network, Studies in Computational Intelligence, Springer-verlag, 149 : 237-246

[8] Chandramathi, Anand, Ganesh, Sriraman and Velmurugan, 2007. Energy Aware Optimal Routing for Wireless Sensor Networks. Journal of Computer Science, 3: 836-840. DOI: 10.3844/jcssp.2007.836.840. 
International Journal of Distributed and Parallel Systems (IJDPS) Vol.3, No.1, January 2012

\section{EXAMPLES OF RELATED PUBLISHED MATERIAL}

[9] Honggang Wang, Dongming Peng, Wei Wang and Hamid Sharif, 2007. Cross Layer Optimization Based on Rate Distribution in Multirate Wireless Sensor Network . Journal of Computer Science, 3: 368-375. DOI: $10.3844 /$ jcssp.2007.368.375.

[10] K. I. Gandhi and P. Narayanasamy, 2011. Mobile Element Scheduling for Efficient Data Collection in Wireless Sensor Networks: A Survey. Journal of Computer Science, 7: 114-119. DOI: 10.3844/jcssp.2011.114.119.

[11]Zurina M. Hanapi, Mahmod Ismail and Kasmiran Jumari, 2009. Priority and Random Selection for Dynamic Window Secured Implicit Geographic Routing in Wireless Sensor Network . American Journal of Engineering and Applied Sciences, 2: 494-500. DOI: 10.3844/ajeassp.2009.494.500.

[12] Kalyani Palanisamy and Chellappan , 2011. Authenticated Broadcast in Heterogeneous Wireless Sensor Networks using Chinese Remainder Theorem Algorithm. Journal of Computer Science, 7: 849-853. DOI: 10.3844/jcssp.2011.849.853.

[13] Shyamala Ramachandran and Valli Shanmugan, 2011. Impact of Sybil and Wormhole Attacks in Location Based Geographic Multicast Routing Protocol for Wireless Sensor Networks. Journal of Computer Science, 7: 973-979. DOI: 10.3844/jcssp.2011.973.979.

[14]T. V.P. Sundararajan, Thiyaneswaran Manoharan and Abinaya Rajendran, 2011. Performance Analysis of Congestion Reduction Routing in Wireless Sensor Networks. Journal of Computer Science, 7: 1011-1019. DOI: 10.3844/jcssp.2011.1011.1019.

[15] A. Balamurugan and T. Purushothaman, 2011. Coverage Eligibility Rule based Coverage Maintenance Protocol for Energy Conservation in Wireless Sensor Networks. Journal of Computer Science, 7: 1545-1553. DOI: 10.3844 jcssp.2011.1545.1553. 